Sensi tivity i mprovement of

si I i con- on- i nsul at or phot odi ode by gol d nanoparticl es wi th substrate bi as control

\begin{tabular}{|c|c|}
\hline 著者 & $\begin{array}{l}\text { Ono At sushi, Nat suo Yuki, Sat oh Hi roaki, } \\
\text { I nokawa Hi r oshi }\end{array}$ \\
\hline $\begin{array}{l}\mathrm{j} \text { our nal or } \\
\text { publ i cat i on } \mathrm{title}\end{array}$ & Appl i ed Physi cs Letters \\
\hline vol une & 99 \\
\hline nunber & 6 \\
\hline page $r$ ange & 062105 \\
\hline year & 2011-08-09 \\
\hline 出版者 & Amer i can I nst i t ute of Physi cs \\
\hline 権利 & ( C) 2011 Amer i can I nst i tute of Physi cs \\
\hline URL & ht t p: //hdl . handl e. net /10297/6172 \\
\hline
\end{tabular}




\title{
Sensitivity improvement of silicon-on-insulator photodiode by gold nanoparticles with substrate bias control
}

\author{
Atsushi Ono, ${ }^{1, a)}$ Yuki Matsuo, ${ }^{2}$ Hiroaki Satoh, ${ }^{2}$ and Hiroshi Inokawa ${ }^{2}$ \\ ${ }^{1}$ Division of Global Research Leaders, Shizuoka University, 3-5-1 Johoku, Naka-ku, Hamamatsu \\ 432-8561, Japan \\ ${ }^{2}$ Research Institute of Electronics, Shizuoka University, 3-5-1 Johoku, Naka-ku, Hamamatsu \\ 432-8011, Japan
}

(Received 11 May 2011; accepted 13 July 2011; published online 9 August 2011)

\begin{abstract}
The sensitivity of silicon-on-insulator (SOI) lateral p-n junction photodiode was enhanced by attaching gold $(\mathrm{Au})$ nanoparticles. This was confirmed by comparing I-V characteristics with and without Au nanoparticles at various substrate voltages. Twofold enhancement was attained in the visible wavelength region when the substrate was biased to positive. The substrate bias changed the area of depletion layer in SOI, and the light scattering by Au nanoparticles effectively enhanced the sensitivity when the area of depletion layer was small. (C) 2011 American Institute of Physics. [doi:10.1063/1.3622650]
\end{abstract}

Silicon-on-insulator (SOI) technology has attracted much attention for years in the field of integrated circuits. ${ }^{1,2}$ It is useful in reducing the parasitic capacitance to realize high-speed operation and low-power consumption. Dielectric device isolation with SOI enables smaller isolation width (i.e., higher integration density), latch-up-free operation, integration with high-voltage devices, etc. Moreover, the use of thin-film SOI is considered as a major method to alleviate the short-channel effect in the future scaled-down transistors. $^{3}$

Although SOI has many advantages, integrated photodetectors tend to suffer from poor sensitivity due to the small silicon thickness for light absorption. In order to address this issue, use of metallic nanoparticles has been attempted. ${ }^{4-7}$ In 1996, Stuart and Hall proposed and demonstrated the enhanced light absorption by the interaction between metal islands formed by vacuum evaporation and the SOI waveguide. ${ }^{4}$ The metal islands of silver (Ag), gold (Au), and copper $(\mathrm{Cu})$ deposited on the $0.16 \mu \mathrm{m}$ thick SOI enhance photocurrent at the wavelength of $800 \mathrm{~nm}$. The enhancement effect on light absorption by metallic nanoparticles has also been applied to bulk Si photodiodes. ${ }^{8,9}$ They demonstrated the localized enhancement of optical absorption in the semiconductor via excitation of surface plasmon resonances in the proximate metal nanopartcicles.

In this Letter, we report the enhanced sensitivity of SOI photodiodes by $\mathrm{Au}$ nanoparticles with special emphasis on the substrate bias effect. The photosensitive area, i.e., the depletion region in the SOI, is varied by the substrate bias, and the sensitivity enhancement by the Au nanoparticles is also correlated to the substrate bias.

Figure 1(a) shows the schematic of the SOI photodiode with Au nanoparticles. This is a lateral $\mathrm{p}^{+} \mathrm{p}^{-} \mathrm{n}^{+}$junction photodiode on a buried oxide (BOX) layer with a thickness of $400 \mathrm{~nm}$. SOI of $95 \mathrm{~nm}$ thick is passivated by the stack of $5 \mathrm{~nm}$ thick sputtered silicon nitride $\left(\mathrm{SiN}_{\mathrm{x}}\right)$ on $5 \mathrm{~nm}$ thick thermal silicon dioxide $\left(\mathrm{SiO}_{2}\right)$. The impurity concentration

\footnotetext{
${ }^{a)}$ Electronic mail: daono@ipc.shizuoka.ac.jp. Telephone: 81-53-478-1370.
}

in $\mathrm{p}^{-}$area of SOI is less than $1 \times 10^{15} \mathrm{~cm}^{-3}$, and one in the $\mathrm{p}^{-}$substrate is about $1 \times 10^{15} \mathrm{~cm}^{-3}$. Au nanoparticles with the diameter of $20 \mathrm{~nm}$ are immobilized on $\mathrm{SiN}_{\mathrm{x}}$ by silane coupling treatment. The anode is connected to the ground. The cathode and the substrate biases are varied between -1 and $1 \mathrm{~V}$ and between -10 and $10 \mathrm{~V}$, respectively. Figure 1(b) shows the optical dark-field image of fabricated SOI photodiode with Au nanoparticles. Mercapt-methyl-trimethoxy-silane (MMTS) is used as an anchor to attach Au nanoparticles on the photodiode. The fabricated SOI photodiode is immersed in a $1 \mathrm{M}$ methyl-isobutyl-ketone solution of MMTS for $1 \mathrm{~h}$. The device is rinsed with ethanol and distilled water and dried with a nitrogen gas. Then, it is dipped into $\mathrm{Au}$ colloidal solution with $\mathrm{Au}$ diameter of $20 \mathrm{~nm}$ and density of $7 \times 10^{11}$ particles/ml, supplied by British BioCell International for $2 \mathrm{~h}$. The density of the attached
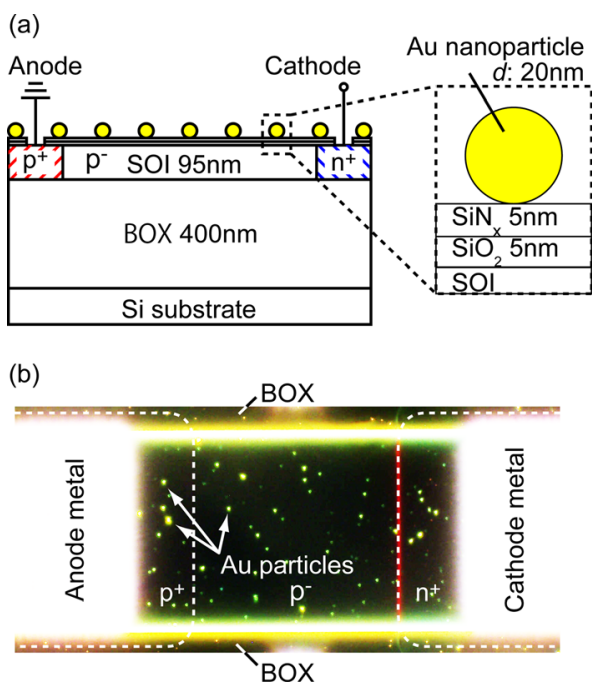

FIG. 1. (Color online) (a) Schematic cross-sectional view of lateral p-n SOI photodiode with $\mathrm{Au}$ nanoparticles. Au nanoparticles are dispersed on the passivation layers of $\mathrm{SiN}_{\mathrm{x}}$ and $\mathrm{SiO}_{2}$. (b) Optical dark-field image of the photodiode with $\mathrm{Au}$ nanoparticles. Bright spots indicate the dispersed Au nanoparticles. Size of the $\mathrm{p}^{-}$area is $50 \mu \mathrm{m} \times 50 \mu \mathrm{m}$. 


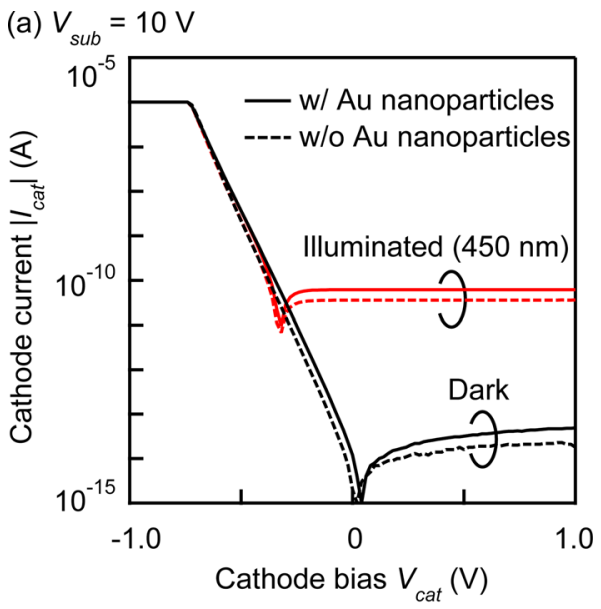

(b) $V_{\text {sub }}=-10 \mathrm{~V}$

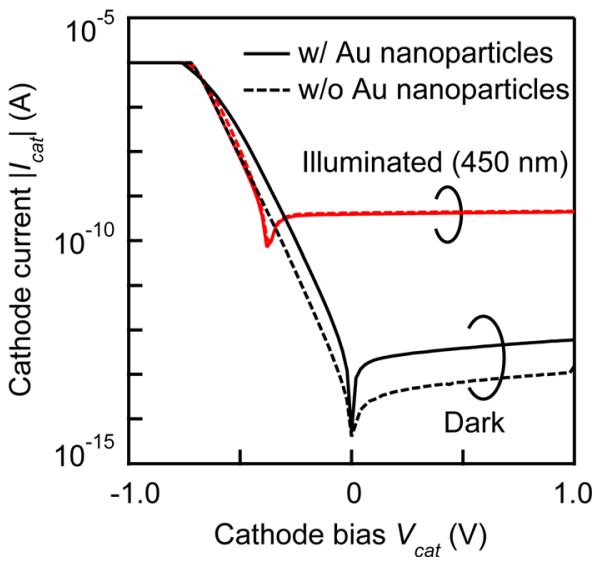

FIG. 2. (Color online) Characteristics of absolute cathode current against cathode bias for substrate biases of (a) 10 and (b) $-10 \mathrm{~V}$, with and without $\mathrm{Au}$ nanoparticles. Incident light is fixed at $\lambda=450 \mathrm{~nm}$. Owing to the presence of Au nanoparticles, photocurrent is augmented at the substrate bias of $10 \mathrm{~V}$.

nanoparticles is $3 \times 10^{6} \mathrm{~cm}^{-2}$ without causing unfavorable surface leakage current.

We measured I-V characteristics of the fabricated device and investigated the changes of the quantum efficiency (QE) by Au nanoparticles dispersed on the SOI photodiode. Figure 2 shows the cathode current as a function of cathode voltage for substrate biases of 10 and $-10 \mathrm{~V}$, with (solid lines) and without (dashed lines) Au nanoparticles. We confirmed typical I-V characteristics of photodiode with relatively small dark current for the active area of $50 \mu \mathrm{m} \times 50 \mu \mathrm{m}$. When light of $60 \mu \mathrm{W} / \mathrm{cm}^{2}$ at the wavelength of $450 \mathrm{~nm}$ is shed on the photodiode, the cathode current is increased by roughly $100 \mathrm{pA}$ for $\mathrm{V}_{\mathrm{sub}}=10 \mathrm{~V}$ and $1 \mathrm{nA}$ for $\mathrm{V}_{\text {sub }}=-10 \mathrm{~V}$. For the substrate bias of $10 \mathrm{~V}$ [Fig. 2(a)], the cathode current is enhanced due to the presence of Au nanoparticles. However, at the substrate bias of $-10 \mathrm{~V}$, the current is nearly the same regardless of the presence of the Au nanoparticles as shown in Fig. 2(b). It is considered that the enhancement by the $\mathrm{Au}$ nanoparticles is related to the depletion area in SOI as will be discussed later.

We evaluated the spectra of external QEs and the ratio of enhancement by the Au nanoparticles as shown in Fig. 3 . The external QE with Au nanoparticles is generally larger than that without them in the visible wavelength at the sub-
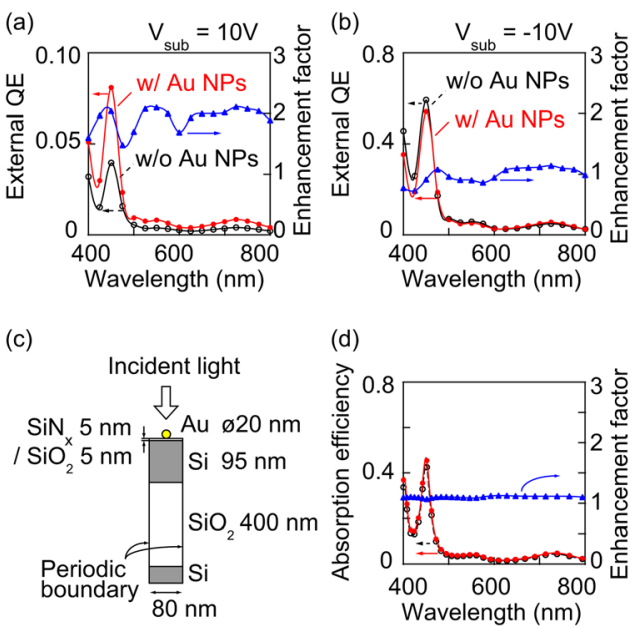

FIG. 3. (Color online) Spectra of external quantum efficiency for substrate biases of (a) 10 and (b) $-10 \mathrm{~V}$, with (filled circles) and without (open circles) Au nanoparticles, and the enhancement factor (triangles). (c) Simulation model of device structure for FDTD method. (d) Simulation result of the absorption efficiency in SOI and enhancement factor with respect to the wavelength.

strate bias of $10 \mathrm{~V}$ (Fig. 3(a)). The enhancement factors are about two. On the other hand, the external QE is almost the same at the substrate bias of $-10 \mathrm{~V}$ [Fig. 3(b)]. The peak at the wavelength of $450 \mathrm{~nm}$ is caused by the multiple reflections and interference effect inside the SOI. Figure 3(c) shows the model for finite-difference time-domain (FDTD) simulation. The model of SOI structure has the same size parameters as in the fabricated device. Periodic boundary conditions are set on the both sides of the SOI structure, and the normal incident light of impulse is irradiated. Spectra of the transmitted powers at the top and bottom interfaces of SOI are obtained by fast Fourier transformation and subtracted. The resultant spectra of the absorption efficiency in SOI and the enhancement factor are shown in Fig. 3(d), which is in good agreement with the experimental data when the substrate is biased to $-10 \mathrm{~V}$ [Fig. 3(b)]. Since the simulation model assumes periodic (i.e., infinitely large) structure, the corresponding experimental condition of $-10 \mathrm{~V}$ substrate bias realizes the case where the light-sensitive depletion region spreads widely over the $\mathrm{p}^{-}$area. On the contrary, the substrate bias of $10 \mathrm{~V}$, which shows much smaller QEs, should correspond to the case where the depletion region is shrunk, suggesting that the enhanced sensitivity by the Au particles [Fig. 3(a)] is the local effect that cannot be reproduced by the infinitely large structure. The most probable cause of the sensitivity enhancement is the contribution of the light scattered by the surrounding Au nanoparticles and absorbed efficiently in the SOI due to the long light path in the lateral direction. Note that even in the case of simulation for the infinitely large structure, small degree of enhancement can be observed, which can be attributed to the light trapping and scattering effects by the Au nanoparticles on top of the SOI photodiode. ${ }^{10}$

Figure 4(a) shows the external QEs and the enhancement factor by Au nanoparticles with respect to the substrate bias from -10 to $10 \mathrm{~V}$. The external QE decreases as the substrate bias increases and becomes constant at the bias over $0 \mathrm{~V}$. This result clearly indicates that the area of the 


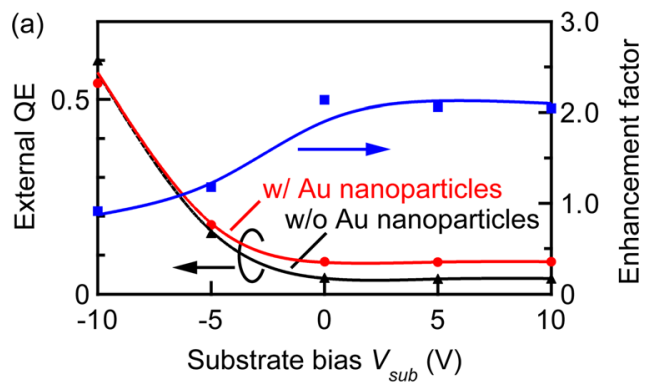

(b)

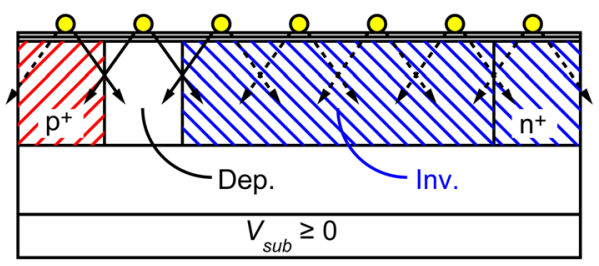

(c)

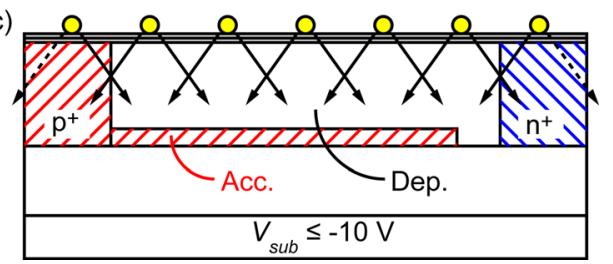

FIG. 4. (Color online) (a) Substrate bias dependences of the external QE with (circles) and without (triangles) Au nanoparticles, and of the enhancement factor (squares). A model to explain the substrate bias dependence of the quantum efficiency and the enhancement factor for substrate biases of (b) $\geq 0$ and (c) $\leq-10 \mathrm{~V}$. Solid arrows indicate the scattered light into the depletion region, which contributes to the photocurrent.

depletion layer in SOI is changed by the substrate bias. On the other hand, the enhancement factor increases as the substrate bias increases and is saturated to twofold above $0 \mathrm{~V}$. Figures 4(b) and 4(c) show a model to explain the substrate bias dependence of QE and the enhancement factor. When the substrate is biased to zero or positive voltage [Fig. 4(b)], $\mathrm{p}^{-}$SOI area is mostly inverted (Inv) due to the presence of the positive fixed charge in the surface nitride/oxide layers and the small dopant concentration in the $\mathrm{p}^{-}$SOI area. Note that in general, the surface of lightly-doped p-type silicon tends to be inverted. ${ }^{11}$ The depletion region (Dep), i.e., lightsensitive region, is localized near the $\mathrm{p}^{+}$region, leading to the small QE. For negative substrate bias [Fig. 4(c)], bottom interface of SOI is accumulated (Acc) with holes, and deple- tion layer (Dep) is formed in a large area, resulting in the enhanced QE. The $\mathrm{p}^{-}$SOI area is completely depleted because the impurity concentration is less than $1 \times 10^{15}$ $\mathrm{cm}^{-3}$, which is sufficiently small to make the 95-nm SOI depleted. ${ }^{12}$ When Au nanoparticles are dispersed, they scatter light to adjacent areas and works as an antenna with an extended light collecting area. Since the reach of light collection is limited, effect of the "antenna" is larger for the small light-sensitive area in the case of zero or positive substrate bias [Fig. 4(a)].

In conclusion, we demonstrated that the quantum efficiency of SOI photodiode was enhanced by attaching $\mathrm{Au}$ nanoparticles on the sample surface including the photodiode. The area of depletion layer in SOI was modulated by the substrate bias, and the smaller area led to the larger enhancement factor. The enhancement mechanism was explained based on the light scattering by the surrounding Au nanoparticles and the efficient absorption of the scattered light by the lateral SOI photodiode. Ensuring this assumption, the distributions of depletion and inversion region should be analyzed by device simulation. Since it is considered that the other improvement mechanism such as affects of week coupling between Au nanoparticles and SOI, further extensive measurements of the device and simulations are required.

${ }^{1}$ J.-P. Colinge, Silicon-On-Insulator Technology: Materials to VLSI, 2nd ed. (Kluwer Academic, Massachusetts, 1997).

${ }^{2}$ S. Cristoloveanu and S. S. Li, Electrical Characterization of Silicon-OnInsulator Materials and Devices (Kluwer Academic, Massachusetts, 1995).

${ }^{3}$ See http://www.itrs.net/Links/2009ITRS/2009Chapters_2009Tables/2009_ ExecSum.pdf for more information about International Technology Roadmap for Semiconductors (ITRS).

${ }^{4}$ H. R. Stuart and D. G. Hall, Appl. Phys. Lett. 69, 2327 (1996).

${ }^{5}$ H. R. Stuart and D. G. Hall, Phys. Rev. Lett. 80, 5663 (1998).

${ }^{6}$ H. R. Stuart and D. G. Hall, Appl. Phys. Lett. 73, 3815 (1998).

${ }^{7}$ S. H. Lim, D. Derkacs, and E. T. Yu, J. Appl. Phys. 105, 073101 (2009).

${ }^{8}$ D. M. Schaadt, B. Feng, and E. T. Yu, Appl. Phys. Lett. 86, 063106 (2005).

${ }^{9}$ S. H. Lim, W. Mar, P. Matheu, D. Derkacs, and E. T. Yu, J. Appl. Phys. 101, 104309 (2007).

${ }^{10}$ H. A. Atwater and A. Polman, Nat. Mater. 9, 205 (2010).

${ }^{11}$ E. H. Nicollian and J. R. Brews, MOS (Metal Oxide Semiconductor) Physics and Technology (Weily, New York, 1982).

${ }^{12}$ H. K. Lim and J. G. Fossum, IEEE Trans. Electron Devices ED-30, 1244 (1983). 